\title{
Educating Consumers and Producers of Data: Review of Making Sense of Numbers by Jane E. Miller (2022)
}

Andrew J. Miller

Belmont University, andrew.miller@belmont.edu

Follow this and additional works at: https://digitalcommons.usf.edu/numeracy

Part of the Other Mathematics Commons

\section{Recommended Citation}

Miller, Andrew J.. "Educating Consumers and Producers of Data: Review of Making Sense of Numbers by Jane E. Miller (2022)." Numeracy 15, Iss. 1 (2022): Article 6. DOI: is https://doi.org/10.5038/ 1936-4660.15.1.1411 


\title{
Educating Consumers and Producers of Data: Review of Making Sense of Numbers by Jane E. Miller (2022)
}

\begin{abstract}
Author Jane E. Miller has brought her considerable experience writing and teaching about numerate communication to a new textbook, Making Sense of Numbers. Miller uses clear prose, timely and authentic examples, and thought-provoking exercises to educate the next generation of consumers and producers of data, students in introductory quantitative reasoning, research methods, or data analysis courses. While the textbook does not fit the mold of a "typical" quantitative literacy course, creative instructors may find ways to use it in innovative quantitative literacy, data literacy, or introductory data science courses.
\end{abstract}

\section{Keywords}

quantitative literacy, research methods, data literacy, textbook

\section{Creative Commons License}

(c) (i) (8)

This work is licensed under a Creative Commons Attribution-Noncommercial 4.0 License

\section{Cover Page Footnote}

Andrew J. Miller is Professor of Mathematics at Belmont University. 
Quantitative literacy educators face two common and related questions: First, "What is quantitative literacy?" Second, "What is a quantitative literacy course?" For better or worse, particularly for multi-section general education courses intended to be taken by large numbers of students, the answer to the second question is often limited by the textbooks available. Thus, the question of what constitutes a quantitative literacy course often reduces to the question of what textbook to adopt. This was certainly the case at my institution when we recently implemented a university-wide quantitative reasoning requirement.

A biased sampling of the quantitative literacy textbook market indicates a range of possible QL courses. There are topical approaches, either focusing on case studies from the media (Madison et al. 2012) or common everyday contexts (Bolker and Mast 2021). Instead, one might build on a key idea, such as ratios, and illustrate its use in a number of quantitative contexts (Gaze 2020). Other courses follow what sometimes seems to be a "standard" list of units: percentages and ratios; linear and exponential models; personal finance; basic probability and statistics; and, perhaps, some voting theory, art, geometry, or fair division (Bennett and Briggs 2019; Denley and Hall 2020; COMAP 2022).

Enter Making Sense of Numbers, by Jane E. Miller, which presents a different approach, suggesting a different kind of entry-level quantitative course. Miller's textbook helps students make sense of numbers in at least two ways: "make sense" as in understand what they read, and "make sense" as in communicate clearly about data. The depth to which Miller discusses this second sense-clear communication - is unusual for QL textbooks but natural for her. Miller has previously written two books on writing about numbers (Miller 2015; 2013), and themes from these books appear throughout Making Sense of Numbers. These themes include an emphasis on "the W's" (who, what, where, when, which; and honorary members so what, how, and how many), focusing on direction and magnitude of quantitative trends, and a "generalization, example, exception" template for describing relationships between quantities.

In the introduction, Miller explains how the goal of the text differs from books like those sampled above: Her aim is to help students succeed at a variety of "quantitative research tasks," including "us[ing] numeric facts to make decisions ... doing simple comparisons using numeric facts from books, reports, or websites ... analyzing a set of data that someone else has collected ... collecting quantitative data ... [and] communicating numeric results" (3). The framing of these QL contexts as "research tasks" reflects Miller's background in social science research methods and is an interesting perspective to bring to a QL course. ${ }^{1}$ This perspective holds throughout the text, which most often presents data in the context of a

\footnotetext{
${ }^{1}$ Editor's Footnote: Readers with a background or interest in numeracy in the context of the social sciences might find Michael Anthony Lewis' Social Workers Count: Numbers and Social Issues of interest. Lewis was reviewed in Numeracy, Volume 14, Issue 1, January 2021.
} 
research study. This context means that the text is aiming at a different audience than what QL educators may think of as "typical."

The text is organized into five parts. Two introductory chapters in Part I are followed by Part II, consisting of three chapters on topic, measurement, and context (i.e., the what, how, when, where, and who of quantitative studies). Part III presents two chapters on effective tables and charts, extending and adapting similar chapters from The Chicago Guide to Writing about Numbers (Miller 2015, hereafter referred to as Writing about Numbers). Part IV includes three chapters with more details on the mathematics and statistics behind many numbers reported in studies and the media. Here is where we find discussions of more familiar QL topics like ratios, rates, percentages, mean, median, mode, and data distributions. Part V becomes more technical, discussing bias, causality, and inferential statistics. Part VI, "Pulling it All Together," concludes the text with two chapters: The first, on communicating quantitative research, is largely a summary of her aforementioned Writing about Numbers. The last chapter recapitulates the textbook's themes and introduces a new one: the need to understand the practical importance of numbers one consumes, produces, or disseminates. There are three appendices as well, on creating new variables, sampling weights, and more details on inferential statistics.

An introductory-level textbook lives or dies by its exercises, and the exercises in Making Sense of Numbers are excellent. Each section includes individual and group exercises, and these are further subdivided into categories of "quantitative research in everyday life," "interpreting research," "planning research," and "analyzing data." Exercises frequently refer to authentic research studies or external sources. They deal with a wide range of topics, including many exercises that connect text concepts to quantitative aspects of COVID-19.

When students are directed in an exercise to find information from their personal experience or individual research, the instructions and scope are clear. In addition, the exercises in the text aim at the upper levels of Bloom's taxonomy, and my impression is that they would be effective in generating rich small-group and full-class discussions. For example, the exercises in Chapter 10, "Distributions and Associations," include one exercise on reading a chart; three exercises on reading and interpreting research studies or journal articles; and six exercises that ask students to collect and analyze actual data (such as on the distribution of colors in a bag of M\&Ms, or the association between age and coffee-drinking among their classmates.) For comparison, in a similar section in Using and Understanding Mathematics (Bennett and Briggs 2019), most exercises involve calculating measures of center and variation from provided data; five out of 36 ask students to use a data set they find on their own; and none ask students to collect their own data. (Miller provides examples of basic exercises in a test bank on a supplemental website.) Miller's exercises include threads that return from chapter to chapter, such as a sequence of exercises that asks students to choose a study from the website of 
the Inter-University Consortium for Political and Social Research and look into different aspects of that study as new topics arise in the text.

Another strength of the text is its choice of examples. Almost all examples use actual, not hypothetical, data and research studies. They cover a wide range of topics, including crime statistics (hate crimes, categories of felony), personal health (COVID 19), political science (definitions of class), psychology (the definition of love), and sports (tennis scoring, team rankings). Tables and charts are, almost without exception, impeccably designed and annotated. There are occasional problems with the formatting of the tables in the e-textbook I reviewed, but based on the electronic tables provided in supplemental material, I believe this issue arose as an artifact from the conversion from print to digital. I will note two uncharacteristic errors: One is found on p. 81, in an example about fuel economy: the units of fuel economy in Britain are listed as $\mathrm{L} / \mathrm{km}$, when the correct units are $\mathrm{L} / 100 \mathrm{~km}$. Another error occurs in Table 5B on p. 110: students are asked to choose the plausible value for the 2017 population of China from 4.5 million, 4.5 billion, and 45 billion, none of which are correct.

The end of each chapter includes a short list of contemporary, accessible recommended readings. Each chapter also includes a clear detailed text description of all images and figures for accessibility. An accompanying website contains supplementary material such as slides, figures, and a test bank (including multiple choice, short answer, and essay assessments appropriate for quizzes or tests). The website for Miller's Writing about Numbers includes datasets for some of the examples in that text; the website for Making Sense of Numbers does not, and I think such datasets would make a useful addition to the supplementary material.

Chapters 7 and 8, on creating effective tables and charts, are two of my favorite chapters. Ever since I first read Miller's Writing about Numbers, I've incorporated her guidance on tables and charts in advice to my students. One of Miller's most useful core principles in this context is to always make a table or chart selfcontained so that it can be read and understood independently from the text. I also wish every student would read and absorb Miller's advice on reporting an appropriate number of digits for a measurement or analysis. In the chapters on tables and charts in Making Sense of Numbers, Miller repeats and extends her advice from Writing about Numbers, going into much more detail than other introductory texts. For example, in the chapter on tables, Miller discusses details such as indentation, panels, ordering for meaning, and three-way tables. In the chapter on charts, she includes lessons from research on visual perception and cognitive load and their implications for chart design. She also discusses several less common (at least in my experience) chart styles like dumbbell plots, lollipop charts, slopegraphs, and heat maps. The material on charts in Making Sense of Numbers goes beyond her earlier work by including guidance on less formal "data 
visualizations," including interesting examples such as text visualizations for effectively presenting a single value.

I also quite liked the concluding chapter, Chapter 15. Despite its title of "The Role of Research Methods in Making Sense of Numbers," I believe this chapter would be useful in any QL course. After summarizing the importance of "the w's" in understanding and contextualizing quantitative data, Miller spends the bulk of the chapter on ways to evaluate the "practical importance"- the "so what?"-of data. Part of this discussion is a good introductory-level discussion of recent conversations in the statistical literature about the use and misuse of statistical significance and "p-hacking." This discussion pairs well with a section in Chapter 13 on "Drawbacks of Traditional Hypothesis Testing" and is the first time I've seen these issues presented in an introductory textbook. Miller also presents an excellent discussion of "relations among dimensions of importance," connections (and nonconnections) between practical importance, effect size, statistical significance, causality, and validity.

Miller also does well presenting less distinctive material, such as in Chapter 9 on comparisons and in Chapter 10 on distributions and associations. Her discussion of percentages, percentage change, percentage difference, and percentile is clear if a little dense, and I would be happy to use her discussion of mean, median, mode, shape, variance, standard deviation, and z-scores in any class. Potential adopters of this text should note that the presentation of these topics is shorter than many other introductory level texts.

For the most part, Miller's explanations are clear, brief, and precise. Perhaps they are a little too brief; the text is dense with terminology. For example, Chapter 2 has 46 newly defined technical terms - from "bivariate association" to "number" to "variable"-in 20 pages, 2.6 new terms per page. By comparison, Chapter 5, "Exploring Data: Distributions," in For All Practical Purposes (COMAP 2022) defines less than one term per page (33 terms in 34 pages). Much of the terminology is excessively technical for a QL audience and reflects the author's years of experience teaching research methods courses. For example, discussions of "level of measurement" arise throughout the text. I expect that social science or statistics students will benefit from the detailed discussion between nominal, ordinal, interval, and ratio variables, but the importance of the distinction may be lost on a general quantitative literacy audience.

The clarity of the vast majority of Miller's text makes the few unclear sections stand out even more. To return to "interval" and "ratio" variables, Miller makes clear that an interval variable is one that has no natural zero point and a ratio variable is one that does but fails to connect these definitions to the names "interval" and "ratio." This omission is a bit surprising because her earlier Writing about Numbers clearly explains that subtraction makes sense for the first kind of variable 
(producing an "interval"), but division (producing a "ratio") only makes sense for the second kind.

Other questions or inconsistencies from the text include the following: On p. 83 , giving different quiz questions to a class of students is described in the context of inter-item reliability, but this practice seems to me an example of alternate forms reliability. On p. 166, a pie chart is presented for the "Distribution of languages spoken at home," with categories "Speak only English," "Spanish," "Other IndoEuropean," "Asian/Pacific Island," and "Other." These categories do not appear to be mutually exclusive, so I am not sure why a pie chart is appropriate. On p. 240, percentile is defined: "The kth percentile is the value for which $\mathrm{k} \%$ of values in the distribution are at or below that value;" but in an example on that page we are told that "Sophia's height placed her in the 38th percentile, meaning that she was taller than $37 \%$ of girls her age based on an international standard." On p. 241, we are told that subtraction can only be performed on continuous variables; however, I have often seen ranks subtracted, for example to display the number of positions gained or lost over time.

The text is aimed at many different audiences, and Miller describes in the Preface how she would use the text for courses in quantitative reasoning, research methods, statistics or data analysis, or quantitative communication. She conceives her theme of "research" very broadly, writing

Even if you aren't thinking of research as a major focus of your career-what I refer to as "Research with a Capital R" - you will need to conduct research in various ways as you go about your daily life, act as an informed citizen, and perform the functions of your profession. To make sense of all the numbers you run across, you need to know how to use research methods and quantitative reasoning skills to find, analyze, interpret, and communicate numeric information. (xxix)

The text reads as if it were written primarily for a research methods course for students in the social sciences, though, and I think it would be more difficult to use this textbook for some of the other courses mentioned. Many general education quantitative literacy instructors (myself included) might find the focus on reading and interpreting research studies too narrow. I would also revise Miller's suggestion of chapters to use for a quantitative reasoning course. She recommends Chapters 1 through 9 and "perhaps" Chapter 15. This outline commendably includes key chapters that discuss tables, charts, and percentages. However, I would definitely include Chapter 15 and also suggest Chapter 10 (basic statistics) and 11 (bias). On the other hand, I would not include Chapter 5, "Context," whose approach to context (sampling, representativeness, generalization, fallacies of level) does not match what I think of as "context" in a QL course. (I think more about putting numbers into context, like judging the size of a number in comparison to other known or typical values, i.e. big compared to what?). 
I also have different ideas than Miller's for a "data analysis" course, at least an introductory level data analysis course like the one taught at my university. We have recently added an entry-level "Introduction to Data Science" course with no prerequisites, and I taught this course for the first time in Fall 2021. There are few, if any, existing textbooks for such a course, so we are currently using material written by one of our faculty members. As I read Making Sense of Numbers, I would often find discussions that would have benefitted my data science students. Many of the research study topics in the book are ones that we have seen throughout our introductory journey in data science: categorical versus continuous variables, summary statistics, visualizations, making sense of statistical analyses, how variables are defined and how data is collected, etc. There is even a passage in the text about "big data:" what it is and why it's become so prevalent in contemporary society. I could see several chapters of this text being used as a complement to our technical material, including Chapters 1 and 2 (the Introduction), Chapter 4 (measurement), Chapters 6 and 7 (tables and charts), parts of Chapters 9 (comparisons) and 10 (distributions and associations), Chapter 15 ("Pulling It All Together"), and Appendix A ("Why and How to Create New Variables).

There is a lot of material in this text-enough, as Miller points out, for two semesters - so it provides a lot of opportunity for the enterprising and creative instructor to create their own kind of QL course. The text's focus on research methods, the density of technical terminology, and the brevity of some explanations present challenges for its use in "standard" QL courses. However, the discussions are thorough and almost universally clear. Moreover, some presentations, particularly the chapters on producing effective tables and charts, are unique to Miller's work. For those reasons, the text will at minimum be an excellent resource for supplementary material and exercises (even for upper-level courses - all STEM students need the guidance in this text on quantitative communication!), especially for instructors who want to prepare students that are able not only to consume data but also to produce it.

\section{References}

Bennett, Jeffrey and William Briggs. 2019. Using and Understanding Mathematics: A Quantitative Reasoning Approach, $7^{\text {th }}$ ed. New York, NY: Pearson.

Bolker, Ethan D. and Maura B. Mast. 2021. Common Sense Mathematics, $2^{\text {nd }}$ ed. Washington, DC: MAA Press. https://doi.org/10.1090/text/063

COMAP. 2022. For All Practical Purposes: Mathematical Literacy in Today's World, $11^{\text {th }}$ ed. Austin, TX: Macmillan Learning.

Denley, Kim and Mike Hall. 2020. Viewing Life Mathematically: A Pathway to Quantitative Literacy. Mount Pleasant, SC: Hawkes Learning. 
Gaze, Eric. 2020. Thinking Quantitatively: Communicating with Numbers, $2^{\text {nd }}$ ed. New York, NY: Pearson.

Madison, Bernard L., Stuart Boersma, Caren L. Diefenderfer, and Shannon W. Dingman. 2012. Case Studies for Quantitative Reasoning: A Casebook of Media Articles, $3^{\text {rd }}$ ed. Boston, MA: Pearson.

Miller, Jane E. 2013. The Chicago Guide to Writing about Multivariate Analysis, $2^{\text {nd }}$ ed. Chicago, IL: The University of Chicago Press. https://doi.org/10.7208/chicago/9780226038193.001.0001

-. 2015. The Chicago Guide to Writing about Numbers, $2^{\text {nd }}$ ed. Chicago, IL: The University of Chicago Press.

-. 2022. Making Sense of Numbers. Thousand Oaks, CA: Sage Publications. 